\title{
Sentence-level quality estimation by predicting HTER as a multi-component metric
}

\author{
Eleftherios Avramidis \\ German Research Center for Artificial Intelligence (DFKI) \\ Language Technology Lab, Berlin, Germany \\ eleftherios.avramidisedfki.de
}

\begin{abstract}
This submission investigates alternative machine learning models for predicting the HTER score on the sentence level. Instead of directly predicting the HTER score, we suggest a model that jointly predicts the amount of the 4 distinct postediting operations, which are then used to calculate the HTER score. This also gives the possibility to correct invalid (e.g. negative) predicted values prior to the calculation of the HTER score. Without any feature exploration, a multi-layer perceptron with 4 outputs yields small but significant improvements over the baseline.
\end{abstract}

\section{Introduction}

Quality Estimation $(\mathrm{QE})$ is the evaluation method that aims at employing machine learning in order to predict some measure of quality given a Machine Translation (MT) output (Blatz et al., 2004). A commonly-used subtask of $\mathrm{QE}$ refers to the learning of automatic metrics. These metrics produce a continuous score based on the comparison between the MT output and a reference translation. When the reference is a minimal post-edition of the MT output, the quality score produced is intuitively more objective and robust as compared to other QE subtasks, where the quality score is assigned directly by the annotators. In that case, the score is a direct reflection of the changes that need to take place in order to fix the translation. HTER (Snover et al., 2009) is the most commonly used metric as it directly represents the least required post-editing effort.

In order to predict the results of an automatic metric, QE approaches use machine learning to predict a model that associates a feature vector with the single quality score. In this case the statis- tical model treats the automatic metric as a black box, in the sense that no particular knowledge about the exact calculation of the quality score is explicitly included in the model.

In this submission we aim to partially break this black-box. We explore the idea of creating a QE model that does not directly predict the single HTER score, but it jointly predicts the 4 components of the metric, which are later used for computing the single score. This way, the structure of the model can be aware of the distinct factors that comprise the final quality score and also potentially learn the interactions between them. Hence, the focus of this submission will remain on machine learning and there will not be exploration in terms of features. In Section 2 we briefly introduce previous work, in Section 3 we provide details about the method, whereas the experiment results are given in Section 4. In Section 5 we describe the models submitted at the shared-task and we explain why they differ from our best models. Finally, in Section 6 we present the conclusions and some ideas for future work.

\section{Previous work}

The prediction of HTER first appeared as a means to estimate post-editing effort (Specia and Farzindar, 2010). Bypassing the direct calculation of HTER was shown by Kozlova et al. (2016), who had positive results by predicting BLEU instead of HTER. Predicting the HTER score with regards to post-editing operations, such as re-ordering and lexical choices, has been done by adding the relevant features in the input (Sagemo and Stymne, 2016), whereas Tezcan et al. (2016) use the wordlevel quality estimation labels as a feature for predicting the sentence-level score. To the best of our knowledge, all previous work used a model to directly predict a single HTER score, in con- 
trast to Avramidis (2014), which trained one separate model for every HTER component and used the 4 individual predictions to calculate the final score, albeit with no positive results. In our work we extend that, by employing a more elegant machine learning approach that predicts four separate labels for the HTER components but through a single model.

\section{Methods}

\subsection{Machine Learning}

The calculation of HTER is based on the count of 4 components, namely the number of insertions, deletions, substitutions and shifts (e.g. reordering) that are required for minimally post-editing a MT output towards the correct translation. The final HTER score is the total number of editing operations divided by the number of reference words.

$$
\text { HTER }=\frac{\text { \#insertions }+ \text { \#dels }+ \text { \#subs }+ \text { \#shifts }}{\text { \#reference words }}
$$

We are here testing 4 different approaches to the prediction of HTER:

1. Baseline with single score: the baseline system of the WMT17 shared task using SVM regression (Basak et al., 2007) to directly predict the HTER score.

2. Combination of $4 \mathrm{SVM}$ models $(4 \times \mathrm{SVM})$ : this is following Avramidis (2014) so that it produces 4 separate SVM regression models that predict the amount of post-editing operations (insertions, deletions, substitutions and shifts respectively). Then, HTER is computed based on the 4 separate outputs (Equation 1).

3. Single-output perceptron (MLP): a multilayer perceptron is trained to predict the HTER score

4. Multi-output perceptron (MLP4): a multilayer perceptron is trained given the feature set in the input and the counts of the 4 postediting operations as the output labels. Similar to $4 \times \mathrm{SVM}$, the separate predictions are used to compute the HTER score (Equation 1). The perceptron is depicted in Figure 1.

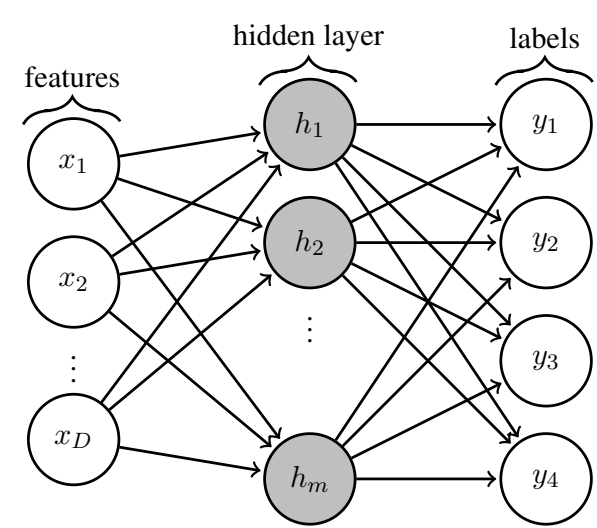

Figure 1: Network graph for the multi-layer perceptron which given the features $x_{1 \ldots D}$ can jointly predict the amount of the post-editing operations $y_{1 \ldots 4}$

In the fist two models, SVM follows the baseline set-up of the WMT17 shared task, using SVM regression with an RBF kernel. The hyperparameters of all three models, including the number of the hidden units of the perceptron, are tuned via grid search on cross-validation with 5 folds over the training data.

\subsection{Normalization of predictions}

Additionally to the separate models, we are testing here some additional normalization on the predicted number of post-editing operations, before it is used to calculate HTER:

i. Integer rounding: although the model was trained using only integers as labels, the regression model resulted into predictions including decimals. By assuming that only an integer amount of post-editing operations should be valid, we round up the post-editing operations to the closest integer.

ii. Trim negative and big numbers: MLP4 may also predict numbers outside the valid integer range, e.g. providing negative numbers or numbers higher than the amount of actual words in the sentence, particularly when features have not been normalized. Here, we trim the invalid values to the nearest integer within the valid range.

\subsection{Optimization measure}

Preliminary experiments indicated that the performance of the MLP4 may vary depending on the optimization metric used for tuning the hyperparameters in a grid search with cross-validation. We 
tested the optimization scoring the folds with $\mathrm{R}^{2}$ and Pearson's rho (which is the official metric of the shared task) in three variations:

a) the $\mathrm{R}^{2}$ of the predicted amount of postediting operations against the golden amount of the post-editing operations

b) the product of 4 rhos (rho edits); each rho evaluating the predictions for one type of post-editing operation (no normalization of predictions) against the golden amount of edits for the same post-editing operation

c) the rho over the final computed HTER (rho HTER) against the golden HTER without any prior normalization of predictions

\section{Experiments}

Here we present the experiments of testing the various machine learning approaches on the development set. After the decisions were taken based on the development set, the models were also applied on the test-sets and the respective results are also given. The performance of the models is measured with Pearson's rho, as this is the basic metric of the WMT17 Shared Task. A test on statistical significance for comparisons is performed with bootstrap re-sampling over 1000 samples. The 4 types of post-editing operations were re-computed with TERCOM on the exact way that the workshop organizers computed the HTER scores. ${ }^{1}$

Similar to the baseline, features values are standardized by removing the mean and scaled to unit variance. Since the experiment is focusing on machine learning, for German-English only the baseline features are used. For English-German, we additionally performed preliminary experiments with the feature-set from Avramidis (2017) including 94 features that improved QE performance for translating into German, generated with the software Qualitative (Avramidis, 2016). The addition of these features did not result into any improvements, so we are not reporting their results during the development phase (see Section 5 for more details). The code for training quality estimation models was based on the software Quest++ (Specia et al., 2015) and Scikit-learn (Pedregosa et al., 2011) ver. 1.18.

\footnotetext{
${ }^{1}$ TERCOM ver. 0.7.25 was downloaded from http: //www.cs. umd.edu/ snover/tercom. The scripts used for running the experiments can be found at https : //github.com/lefterav/MLP 4.
}

The development results concerning the presented methods are given below in this section. The model approaches are tested for both language directions, whereas the experiments on the normalization of the predictions and ML optimization are run only for German-English and these observations are applied to the models for EnglishGerman.

\subsection{Best ML method}

The results concerning the choice of the ML method applied on German-English are shown in Table 1.

\begin{tabular}{lll}
\hline method & \multicolumn{1}{c}{ dev } & \multicolumn{1}{c}{ test } \\
\hline SVM & 0.400 & 0.441 \\
$4 \times$ SVM & 0.392 & 0.409 \\
MLP & $0.447^{*}$ & 0.447 \\
MLP4 & $0.476^{*}$ & $0.475^{* *}$ \\
\hline
\end{tabular}

Table 1: Pearson rho correlation against golden labels concerning the 4 different approaches for predicting HTER for German-English. (*) indicates significant improvement $(\alpha=0.05)$ over the SVM baseline (**) significant improvement over all models

The approach of MLP4 achieves a small but significant improvement over the baseline and the $4 \times$ SVM on the development set. On the development set both MLP and MLP4 beat significantly the baseline, but MLP4 is not significantly better than MLP. Nevertheless, when applied to the testset, the improvement achieved with MLP4 is significant as compared to all other ML methods.

\begin{tabular}{lcrc}
\hline method & dev & test2017 & test2016 \\
\hline SVM & 0.414 & 0.402 & 0.407 \\
$4 \times$ SVM & 0.049 & -0.071 & 0.044 \\
MLP & 0.343 & 0.335 & 0.327 \\
MLP4 & $0.429^{*}$ & 0.412 & 0.412 \\
\hline
\end{tabular}

Table 2: Performance of the 4 different approaches for predicting HTER for English-German (*) indicates a significant improvement $(\alpha=0.1)$ over the baseline

The same approaches show moderate improvements when applied to English-German with the baseline feature set (Table 2). MLP4 achieves higher correlation score than the baseline, but the difference is small and it is significant only for the 
development set. When compared to the other two methods, though, MLP4 achieves a significant improvement. In contrast to the direction GermanEnglish, in English-German the MLP with one output performs worse than the baseline. $4 \times \mathrm{SVM}$ fails to predict HTER as its predictions achieve zero correlation. Since the individual models failed to predict the post-editing operations separately, this may be an indication that among the 4 post-editing operations in English-German there are dependencies which are stronger than the ones in German-English.

\subsection{Normalization of predicted post-editing operations}

The effect of the normalization of the predicted post-editing operations of MLP4, prior to the calculation of the final HTER score, is shown in Table 3 .

\begin{tabular}{lcc}
\hline & dev & test \\
\hline original labels & 0.473 & 0.471 \\
trim & 0.476 & 0.475 \\
round & 0.456 & 0.469 \\
round \& trim & 0.456 & 0.467 \\
\hline
\end{tabular}

Table 3: Performance improvements by introducing rounding and cut-off for the predicted postediting operations (German-English)

The experiment indicates some small improvement when we trim the invalid predicted values, so we use this for all other calculations. Preliminary experiments indicated more significant improvements when the feature values have not been standardized and re-scaled prior to the training.

\subsection{ML optimization}

The effect of using different methods for hyperparameter optimization is show in Table 4.

\begin{tabular}{lcc}
\hline & dev & test \\
\hline $\mathrm{R}^{2}$ & 0.440 & 0.454 \\
rho HTER & 0.431 & 0.457 \\
rho edits & 0.476 & 0.475 \\
\hline
\end{tabular}

Table 4: Experimentation with different optimization measures for defining the perceptron hyperparameters (German-English model)

The product of the 4 rhos, calculated over the 4 types of post-editing operations (rho edits) has slightly better performance than the other scoring methods, nevertheless the difference is not statistically significant. Using these findings just as an indication, we perform all experiments by optimizing the hyperparameters with rho edits.

The optimized hyperparameters for the SVM models are shown in Table 5, whereas the ones for the MLP models are shown in Table 6. All SVMs have an RBF kernel and all MLPs are optimized with adam as a solver. It is noteworthy that for German-English a network topology with multiple hidden layers performed better, which is an indication that the mapping between features and labels in this language pair is much more complex than the one for German-English.

\begin{tabular}{llccc}
\hline langpair & model & $\epsilon$ & $\mathrm{C}$ & $\gamma$ \\
\hline de-en & SVM & 0.1 & 10 & 0.001 \\
& $4 \times$ SVM (ins) & 0.2 & 10 & 0.01 \\
& $4 \times$ SVM (del) & 0.2 & 10 & 0.01 \\
& $4 \times$ SVM (shifts) & 0.2 & 10 & 0.01 \\
& $4 \times$ SVM (subst) & 0.1 & 10 & 0.01 \\
\hline en-de & SVM & 0.1 & 1 & 0.01 \\
& $4 \times$ SVM (ins) & 0.2 & 1 & 0.001 \\
& $4 \times$ SVM (del) & 0.1 & 1 & 0.001 \\
& $4 \times$ SVM (shifts) & 0.1 & 1 & 0.001 \\
& $4 \times$ SVM (subst) & 0.2 & 1 & 0.001 \\
\hline
\end{tabular}

Table 5: Hyperparameters used after the optimization of the SVM models

\begin{tabular}{llcccl}
\hline langp. & model & act. & $\alpha$ & tol. & hidden units \\
\hline de-en & MLP & relu & 0.10 & $10^{-9}$ & $1: 100$ \\
& MLP4 & relu & 0.10 & $10^{-3}$ & $1: 300$ \\
en-de & MLP & $\tanh$ & 0.01 & $10^{-3}$ & $3: 150,75,6$ \\
& MLP4 & $\tanh$ & 0.10 & $10^{-3}$ & $2: 300,150$ \\
\hline
\end{tabular}

Table 6: Hyperparameters and network topology after the optimization of the MLP models

\section{Submission and post-mortem analysis}

Whereas previous sections described a full development phase in order to support the idea of the multi-output MLP, this section is focusing on our exact submission for the Quality Estimation Task of WMT17. Unfortunately, a development issue prior to the submission prevented our experiments from standardizing the feature values and scaling them to unit variance. Since the performance of 
SVM suffers from non-scaled feature values, this led our development phase to proceed by contrasting with a much lower baseline than the one finally provided by the workshop organizers. Non-scaled features and other settings affected also the performance of MLP models, and therefore the scores on our final submissions are significantly lower than the official baseline. The issue became apparent only after the submission, so we then re-computed the all models with standardized and scaled feature values. The results presented in Section 4 are based on these corrected models.

The submitted models used both rounding and trimming of predicted integers (Section 3.2). The MLPs were optimized with an $\alpha=0.01$, tanh as an activation function, and adam as a solver. The German-English model got optimal with 300 hidden units. The English-German was trained using the additional 52 features from Avramidis (2017) which gave good development results only with 3,000 hidden units, which is an indication of overfitting.

\begin{tabular}{lcc}
\hline method & dev & test \\
\hline baseline (ours) & 0.32 & 0.34 \\
MLP4 (submitted) & 0.40 & 0.40 \\
\hline baseline (official) & 0.40 & 0.44 \\
MLP4 (corrected) & 0.48 & 0.48 \\
\hline
\end{tabular}

Table 7: Scores for the submitted models and for their corrected versions after the submission (German-English)

\begin{tabular}{lccc}
\hline method & dev & test2017 & test2016 \\
\hline baseline (ours) & 0.19 & 0.20 & 0.12 \\
MLP4 (submitted) & 0.40 & 0.11 & 0.13 \\
\hline baseline (official) & 0.41 & 0.40 & 0.40 \\
MLP4 (corrected) & 0.43 & 0.41 & 0.41 \\
\hline
\end{tabular}

Table 8: Scores for the submitted models and for their corrected versions after the submission English-German

A comparison of the models developed before the submission and the corrected ones are shown in Tables 7 and 8. The submitted model for German-English was expected to be significantly better than the baseline, whereas the one for English-German with the additional features had strong indications of overfitting and performed indeed poorly at the final test-sets.

The corrected models perform better after scaling is added and the rounding of integers is disabled. The corrected model for English-German converges without overfitting after removing the additional features and adding one more hidden layer. These models, if submitted to the shared task, despite comparing with the baseline, they would still score lower than almost all the others submitted methods. Though, we need to note that this should still be satisfactory, as we did not perform any feature engineering, aiming at confirming our hypothesis for using multi-output models.

\section{Conclusion and further work}

In this submission we investigated the idea of using a multi-layer perceptron in order to jointly predict the 4 distinct post-editing operations, which are then used for calculating the HTER score. The experiments show some small but significant improvements on both the development set and the test-set for German-English, but the same approach showed improvement only on the development set when applied English-German.

Despite not having conclusive results yet, we think that the idea is promising and that further experiments could have positive impact. Concerning the current development, several issues need to be further investigated, such as possible ways to avoid the lack of robustness of the perceptron. Since this work did not focus at feature engineering, further work could profit from introducing features highly relevant to the specific types of post-editing operations, or even upscaling observations from word-level and phrase-level QE. On the machine-learning level, additional hidden layers and more work on the number of hidden units might be of benefit. Finally, evaluation specific to the types of the predicted post-editing operations could provide hints for further improvement.

\section{Acknowledgments}

This work has received funding from the European Union's Horizon 2020 research and innovation program under grant agreement $\mathrm{N}^{\circ} 645452$ (QT21). 


\section{References}

Eleftherios Avramidis. 2014. Efforts on Machine Learning over Human-mediated Translation Edit Rate. In Proceedings of the Ninth Workshop on Statistical Machine Translation. Association for Computational Linguistics, Baltimore, Maryland, USA, pages 302-306. http://www.aclweb.org/anthology/W/W14/W143337.

Eleftherios Avramidis. 2016. Qualitative: Python Tool for MT Quality Estimation Supporting Server Mode and Hybrid MT. The Prague Bulletin of Mathematical Linguistics (PBML) 106:147-158. https://ufal.mff.cuni.cz/pbml/106/art-avramidis.pdf.

Eleftherios Avramidis. 2017. Comparative Quality Estimation for Machine Translation. Observations on machine learning and features. Proceedings of the 20th Annual Conference of the European Association for Machine Translation, The Prague Bulletin of Mathematical Linguistics (108):307-318. https://doi.org/10.1515/pralin-2017-0029.

Debasish Basak, Srimanta Pal, and Dipak Chandra Patranabis. 2007. Support vector regression. Neural Information Processing-Letters and Reviews 11(10):203-224.

John Blatz, Erin Fitzgerald, George Foster, Simona Gandrabur, Cyril Goutte, Alex Kulesza, Alberto Sanchis, and Nicola Ueffing. 2004. Confidence estimation for machine translation. In Proceedings of the 20th international conference on Computational Linguistics (COLING 04). Association for Computational Linguistics, Stroudsburg, PA, USA. https://doi.org/10.3115/1220355.1220401.

Anna Kozlova, Mariya Shmatova, and Anton Frolov. 2016. YSDA Participation in the WMT'16 Quality Estimation Shared Task. In Proceedings of the First Conference on Machine Translation. Association for Computational Linguistics, Berlin, Germany, pages 793-799. http://www.aclweb.org/anthology/W/W16/W162385 .

Fabian Pedregosa, Gael Varoquaux, Alexandre Gramfort, Vincent Michel, Bertrand Thirion, Olivier Grisel, Mathieu Blondel, Peter Prettenhofer, Ron Weiss, Vincent Dubourg, Jake Vanderplas, Alexandre Passos, David Cournapeau, Matthieu Brucher, Matthieu Perrot, and Edouard Duchesnay. 2011. Scikit-learn: Machine Learning in Python. Journal of Machine Learning Research 12:2825-2830.

Oscar Sagemo and Sara Stymne. 2016. The UU Submission to the Machine Translation Quality Estimation Task. In Proceedings of the First Conference on Machine Translation. Association for Computational Linguistics, Berlin, Germany, pages 825-830. http://www.aclweb.org/anthology/W/W16/W162390.
Matthew Snover, Nitin Madnani, Bonnie J Dorr, and Richard Schwartz. 2009. Fluency, Adequacy, or HTER?: Exploring Different Human Judgments with a Tunable MT Metric. In Proceedings of the Fourth Workshop on Statistical Machine Translation. Association for Computational Linguistics, Stroudsburg, PA, USA, StatMT '09, pages 259-268. http://www.aclweb.org/anthology/W/W09/W090x41.

Lucia Specia and Atefeh Farzindar. 2010. Estimating Machine Translation Post-Editing Effort with HTER. AMTA2010 Workshop Bringing MT to the User MT Research and the Translation Industry pages 33-41. http://mt-archive.info/JEC-2010Specia.pdf.

Lucia Specia, Gustavo Paetzold, and Carolina Scarton. 2015. Multi-level Translation Quality Prediction with QuEst++. In Proceedings of ACL-IJCNLP 2015 System Demonstrations. Association for Computational Linguistics and The Asian Federation of Natural Language Processing, Beijing, China, pages 115-120. http://www.aclweb.org/anthology/P154020.

Arda Tezcan, Veronique Hoste, Bart Desmet, and Lieve Macken. 2016. UGENT-LT3 SCATE System for Machine Translation Quality Estimation. In Proceedings of the First Conference on Machine Translation. Association for Computational Linguistics, Berlin, Germany, pages 353-360. http://aclweb.org/anthology/W15-3043 http://www.aclweb.org/anthology/W/W16/W162393. 